\title{
Electroluminescent ceramics excited by low electrical field
}

\author{
Yun Liu ${ }^{\text {a) }}$ and Chao-Nan Xu ${ }^{\text {b),c) }}$ \\ National Institute of Advanced Industrial Science and Technology (AIST, Kyushu), Shuku 807-1, Tosu, Saga, \\ 841-0052, Japan
}

(Received 17 October 2003; accepted 26 April 2004; published online 28 May 2004)

\begin{abstract}
Strong green-light emission occurs in Eu: $\mathrm{SrAl}_{2} \mathrm{O}_{4}$ ceramics and $\mathrm{Eu}: \mathrm{SrAl}_{2} \mathrm{O}_{4} /$ poly(vinylidene fluoride-trifluoroethylene) ( $\mathrm{P}(\mathrm{VDF}-\mathrm{TrFE})$ ) composites when excited by a lower dc or ac voltage. That emission is caused by strong electric-mechanic-optic interaction. The composite shows stronger luminescent emission intensity in comparison to similar ceramics because of an enhanced piezoelectric effect from $\mathrm{P}(\mathrm{VDF}-\mathrm{TrFE})$ - a typical piezoelectric polymer. (C) 2004 American Institute of Physics. [DOI: 10.1063/1.1763223]
\end{abstract}

Most commercial electroluminescent (EL) materials belong to the sulfide family, ${ }^{1-3}$ which generally possesses a broadband gap and requires a high electrical field to excite hot carriers in the luminescent centers. They are also extremely susceptible to environmental changes such as humidity. Those characteristics complicate the device and hinder fabrication. Therefore, although such EL materials are used currently in practical applications, the search continues for new inorganic EL materials to simplify device structure and offer enhanced electroluminescence.

Some EL materials have been developed recently: $\mathrm{SiC}^{4}$ $\mathrm{Y}_{2} \mathrm{O}_{3},{ }^{5}$ porous silica, ${ }^{6}$ and $\mathrm{Gd}_{2} \mathrm{O}_{3}-\mathrm{Ga}_{2} \mathrm{O}_{3} .{ }^{7}$ Most have demonstrated low EL efficiency, but the tendency to substitute for the sulfide family is unequivocal. In addition, most EL devices are made in the form of thin or thick films. No study has sought to develop bulk EL devices with a simple structure. Considering the current situation, this letter pre-

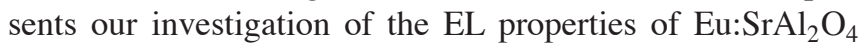
(SAO-E) ceramics and their composites with copolymers. $\mathrm{SrAl}_{2} \mathrm{O}_{4}$ demonstrates high-efficiency photoluminescence (PL) with persistent phosphorescence, ${ }^{8}$ excellent chemical stability, and strong mechanoluminescent properties that yield visible light emission by fracture, friction, and deformation. ${ }^{9-11}$ This latter characteristic can be applied for stress sensors and display devices. ${ }^{12}$ The present study will promote development of an application field for $\mathrm{SrAl}_{2} \mathrm{O}_{4}$ materials.

Strontium aluminates powders were synthesized using a modified base-catalyzed sol-gel process. ${ }^{13}$ Raw materials were aluminum tri-i-propoxide $\left(\mathrm{Al}\left(\mathrm{O}-\mathrm{i}-\mathrm{C}_{3} \mathrm{H}_{7}\right)_{3}\right), \operatorname{Sr}\left(\mathrm{NO}_{3}\right)_{3}$, $\mathrm{Eu}\left(\mathrm{NO}_{3}\right)_{3} \cdot 2.9 \mathrm{H}_{2} \mathrm{O}$, and $N, N$-Dimethylformamide (DMF). The powders were annealed at $1300{ }^{\circ} \mathrm{C}$ for $2 \mathrm{~h}$ in a reducing atmosphere with $5 \% \mathrm{H}_{2}-\mathrm{N}_{2}$ to crystallize and form luminescent centers. Prepared by this process, the powder particles' mean size was $\sim 1-2 \mu \mathrm{m}$ as revealed by scanning electron microscope observation. Resultant powders were mixed thoroughly and pressed into pellets. We obtained crack-free and

\footnotetext{
a) Present address: Research School of Chemistry, Australian National University, Canberra, ACT 0200, Australia.

b) Also at: PRESTO, Japan Science and Technology Agency (JST), 4-18 Honcho Kawaguchi, Saitama, Japan.

${ }^{c)}$ Author to whom correspondence should be addressed; electronic mail: cnxu@aist.go.jp
}

dense ceramic pellets after $24 \mathrm{~h}$ heat-treatment at $1400^{\circ} \mathrm{C}$ in the same reducing atmosphere. Both sides of each ceramic pellet were polished to a smooth and flat surface. The thickness was $1 \mathrm{~mm}$. An ITO transparent electrode was deposited on one side using rf sputtering. The ITO electrode was smaller than the ceramic pellet to allow easy observation of EL phenomena and avoid electrical discharge along the sample brim. A thin conductive probe was pressed onto the ITO surface to obtain an electrical connection with the top electrode. Room-temperature conductive paste was brushed onto the other side of the pellet as a bottom electrode and solidified for 1 day. A Pt wire was embedded into the paste and solidified to provide a connection with the bottom electrode. $\mathrm{SrAl}_{2} \mathrm{O}_{4}$ powders were also mixed with poly(vinylidene fluoride-trifluoroethylene) $(\mathrm{P}(\mathrm{VDF}-\mathrm{TrFE}))$ based on a weight ratio of $\mathrm{Eu}: \mathrm{SrAl}_{2} \mathrm{O}_{4} / \mathrm{P}(\mathrm{VDF}-\mathrm{TrFE})(50 / 50)$ and pressed into pellets $(\phi 15 \times 1 \mathrm{~mm})$ at $130^{\circ} \mathrm{C}$. We also made an EL device for this composite with a similar sandwich structure to that described above.

The EL was characterized by a dc or ac $(60 \mathrm{~Hz})$ electrical power source with an amplifier, and a spectrofluorometer (FP-6500; Jasco Inc.) with an optical fiber attachment. The distance between the EL device surface and the receiving end of the fiber was $15 \mathrm{~mm}$. Electrical resistivity of SAO-E ceramics was measured with an ultrahigh resistance meter (R8340A; Advantest Corp.). The current-voltage property was evaluated by a nanovolt-meter (2182; Keithley Instruments, Inc.). The dielectric property at room temperature was determined by an LCR meter (3522; Hioki E. E. Corp.). The ferroelectric hysteresis loop was observed with an automatic ferroelectricity measurement system (RT6000HVA; Radiant Technologies, Inc.).

Figure 1 presents typical PL and EL emission spectra from SAO-E ceramics and SAO-E/P(VDF-TrFE) composites. They both present a broadband emission with a green color. The PL emission peak of SAO-E ceramics centers at $518 \mathrm{~nm}$ and its EL is at $510 \mathrm{~nm}$; the SAO-E composite shows corresponding features at 516 and $524 \mathrm{~nm}$, respectively. The EL emission wavelength shift is most likely the result of preferred emission from different splitting $5 d$ energy levels under the electrical field. Nonetheless, EL is emitted from the same emission centers of $\mathrm{Eu}^{2+}$ ions as PL: both result from the $5 d \rightarrow 4 f$ transition of $\mathrm{Eu}^{2+}$ ions in $\mathrm{SrAl}_{2} \mathrm{O}_{4} \cdot{ }^{14}$ 


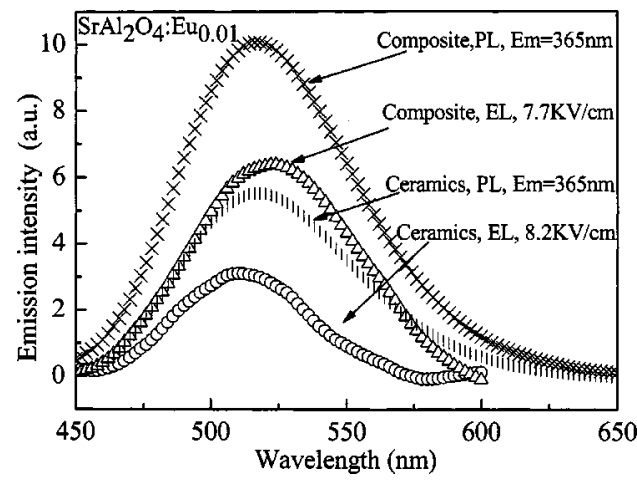

FIG. 1. Photoluminescence (PL) and electroluminescence (EL) spectra of SAO-E ceramics and the SAO-E/P(VDF-TrFE) composite.

Figure 2 shows that the EL of SAO-E can be excited by only a few kilovolts per centimeter. Based on the assumption that light emission is excited by $10 \mathrm{kV} / \mathrm{cm}$, the excited voltage is only $1 \mathrm{~V}$ for SAO-E ceramics with a thickness of 1 $\mu \mathrm{m}$. Existing techniques would allow a useful $0.5-\mu \mathrm{m}$-thick SAO-E film. ${ }^{15,16}$ Therefore, this result has exciting implications for future development of SAO-E thin film EL devices.

Why can this low voltage excite such a strong EL in SAO-E ceramics? Figure 2 shows that the light emission intensity of SAO-E does not increase linearly with increasing electrical voltage. Correlation of EL intensity $\left(B_{\mathrm{el}}\right)$ and voltage $(V)$ can be divided into two stages (see Fig. 2). The $B_{\text {el }}$ increases linearly and slowly at stage I $(0 \leqslant V$ $\leqslant 4.2 \mathrm{kV} / \mathrm{cm})$. It increases steeply, but maintains linearity at stage II $(V \geqslant 4.2 \mathrm{kV} / \mathrm{cm})$. At low voltage (stage I), the current is initially carried by thermally excited carriers contained in the common insulator; thereby, the current-voltage displays an ohmic feature where $B_{\mathrm{el}} \propto V .{ }^{17}$ Electroluminescence in the sulfide semiconductor $\mathrm{ZnS}$ demonstrates a similar property. However, its EL mechanism at high voltage is unsuitable for SAO-E. The EL of $\mathrm{ZnS}$, with an energy gap of $\sim 3.7 \mathrm{eV}^{18}{ }^{18}$ results from excitation of hot carriers under a high electrical field of about $106 \mathrm{kV} / \mathrm{cm} \cdot{ }^{17,18}$ This excitation causes the diversity in its linear ohmic characteristics. However, the $I-V$ curve remains linear for $\mathrm{SrAl}_{2} \mathrm{O}_{4}$, which has an energy gap of $6.4 \mathrm{eV}$. Moreover, it is unreasonable for the $\mathrm{Eu}^{2+}$ luminescent centers to be excited by hot carriers. Electrical properties are investigated in this letter to explore the mechanism of EL in SAO-E.

The dielectric constant of SAO-E is determined to be

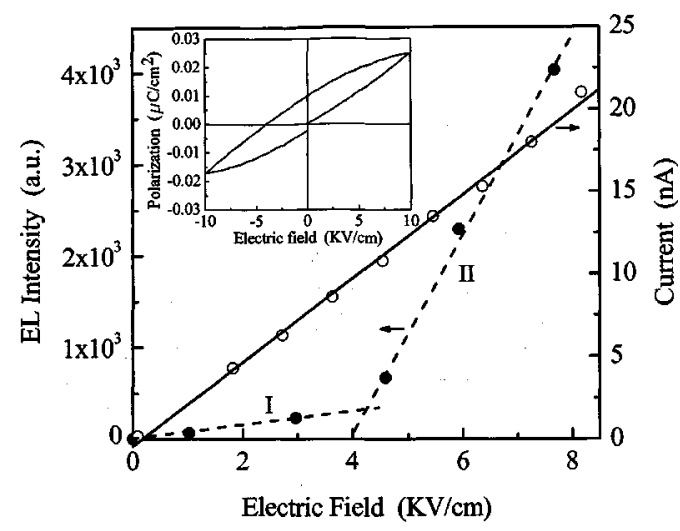

FIG. 2. Relations of EL intensity, current, and voltage in SAO-E ceramics (an inset is its ferroelectric hysteresis loop). trons and holes occurs, this is another possible EL mecha-
Downloaded 01 Oct 2007 to 150.203.35.38. Redistribution subject to AlP license or copyright, see http://apl.aip.org/apl/copyright.jsp only 11 at room temperature. It offers good electrical insulating properties: its electrical resistivity reaches $10^{10} \Omega / \mathrm{cm}$. We further observed the ferroelectric hysteresis loop in SAO-E ceramics (see the inset in Fig. 2). This observation confirms that SAO-E is a ferroelectric ceramic in experimentation. As ferroelectrics must also be piezoelectrics, SAO-E should possess a piezoelectric property. Furthermore, we discovered strong mechanoluminescence in SAO-E ceramics and composites. ${ }^{10,19}$ All of these identify the need to consider the triangular link in mechano-electro-optics. Chandra ${ }^{20}$ studied mechanoluminescent properties of $\mathrm{ZnS}$ and $\mathrm{ZnSe}$, and thereby mathematically described the emission intensity of mechanoluminescence $\left(B_{\mathrm{ml}}\right)$ regarding the strain:

$$
B_{\mathrm{ml}}=\alpha \frac{\partial x}{\partial t}(1-\exp (-t / \tau))
$$

Here, $\alpha$ is a constant associated with the radius of interaction of a dislocation, the dislocation capture probability, and the density of activators. $\partial x / \partial t$ is the strain rate. $\tau$ is the lifetime of the interaction.

The strain $(x)$ created by the piezoelectric effect is generally expressed as

$$
x_{i}=d_{m i} E_{m}=d_{m i} V_{m} / \Theta,
$$

where $d$ is piezoelectric coefficient. $E$ and $V$ are the electric field and voltage, respectively; $\Theta$ is the sample thickness. Assuming that strain occurs in the same direction with the piezoelectric effect, Eq. (2) can be simplified as

$$
x=d V / \Theta,
$$

Therefore,

$$
\frac{\partial x}{\partial t}=\frac{d}{\Theta} \frac{\partial V}{\partial t} .
$$

For dc voltage, initially, $V=a t$, where $a$ is the rate of voltage increase. Consequently, the emission intensity of luminescence depends on the rate of voltage increase. For ac voltage, $V=V_{0} \sin \omega t$ : the emission intensity is modulated by the ac signal. Consequently, it follows that EL in SAO-E results from electro-mechano-optical interaction. The electrical field induces strain, which could stimulate light through a mechanism similar to mechanoluminescence described previously in Ref. 16.

An inset in Fig. 2 demonstrates that the SAO-E has a small polarization electrical field because of its low dielectric constant and high resistivity (corresponding to a low dielectric loss), this agrees with the result that SAO-E ceramics are soft and their strain can be induced by a lower electrical field. ${ }^{21}$ This hysteresis loop shows that a smaller internal electrical field $\left(E_{p i}\right)$ will be built up through this electrical field polarization. In addition, the strong asymmetry hysteresis loop indicates that an internal electrical field $\left(E_{i i}\right)$ had been in the SAO-E sample when the external electrical field was zero. $E_{i i}$ and $E_{p i}$ have the same direction. Thereby, the local electrical field ( $\left.E_{\text {local }}\right)$ in SAO-E ceramics will be enhanced $\left(E_{\text {local }}=E_{p i}+E_{i i}\right)$. Such an electrical field may excite the holes and electrons from corresponding carrier traps and thereby release an emission associated with the $5 d$ $\rightarrow 4 f$ transition of Eu ions when recombination of the elec- 


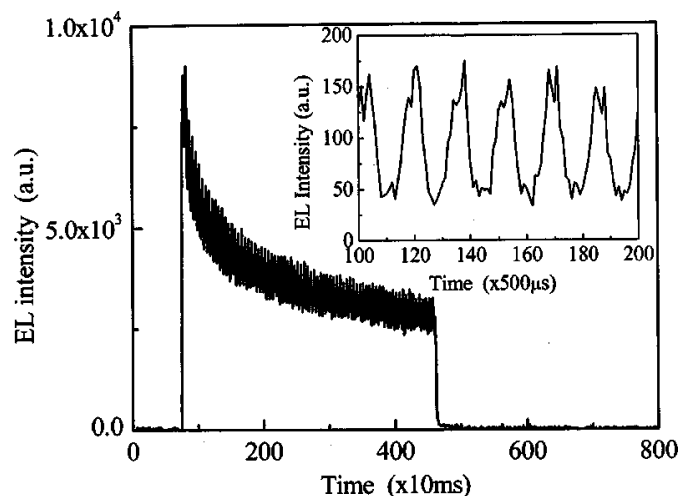

FIG. 3. EL response of the SAO-E composite. The inset presents detail of modulated light emission signals.

nism for SAO-E. As previously reported, the SAO-E ceramics had several carrier traps. Their levels were determined by thermal luminescence techniques to be on the order of 0.2 $\mathrm{eV}^{9,12}$ These shallow traps are inferred to be excited by an electrical field that exceeds the threshold, as in stage II of Fig. 2.

On the other hand, the EL produced by the strainstimulated mechanism can be excited by a lower electric field. As already described, this mechanism is reasonable for the whole range with electric fields, from stage I to stage II (Fig. 2).

Consequently, this work has built up a triangle interaction in mechano-electro-optics. This correlation has been proved in $\mathrm{ZnS}: \mathrm{Mn},{ }^{19}$ where mechanoluminescence is activated by a piezoelectric effect. Similarly, the piezoelectric property bridges the stress and luminescence to create the EL property in SAO-E.

Figure 1 also shows that the relative intensity of EL/PL is higher in the SAO-E composite than in ceramics. This strengthened effect originates from $\mathrm{P}(\mathrm{VDF}-\mathrm{TrFE})$-a wellknown piezoelectric copolymer. Piezoelectrics of $\mathrm{P}(\mathrm{VDF}-$ TrFE) enhance the local internal electrical field by a product effect, thus exciting a stronger light emission. Therefore, the mechanism of electro-mechano-optical interaction explains mechanoluminescent behavior of the SAO-E ceramics and composites.

Light emission intensity declines concomitant with the increasing holding time of the electrical field. Keeping the dc voltage unchanged, the $B_{\text {el }}$ does not decline zero even though the $\partial x / \partial t=0$. This decline results from the especially persistent phosphorous characteristics in SAO-E. The attenuation can be described simply as $y=A+B e^{-x / C}$, where $A, B$, and $C$ are constants with either ac or dc voltage, but the response curve of SAO-E composites shows a modulated light emis- sion under the action of ac voltage, as shown in Fig. 3. The inset demonstrates partial modulated light emission signals, which are both $60 \mathrm{~Hz}$. This phenomenon confirms the above theoretical model. The peak and valley of the sine curve have two smaller modulated peaks, most likely resulting from space charges or dipole reversion.

In conclusion, a strong green-light emission can be observed clearly by the naked eye in both samples. The SAO-E particles are smaller than $2 \mu \mathrm{m}$ in the composite, which allows homogeneous dispersion of ceramic powders into the copolymer matrix. Therefore, EL devices that consist of three layers without an insulating dielectric layer are obtained. Although further research is needed on the profound mechanism of EL and its attenuating behavior, this work has demonstrated that $\mathrm{Eu}: \mathrm{SrAl}_{2} \mathrm{O}_{4}$ ceramics can yield bright electroluminescence. This finding will greatly broaden application of SAO-E based materials.

${ }^{1}$ M. Leskela, Springer Proc. Phys. 38, 204 (1989).

${ }^{2}$ P. M. Fauchet, L. Tsybeskov, S. P. Duttagupta, and K. D. Hirschaman, Thin Solid Films 297, 254 (1997).

${ }^{3}$ K. Tanaka, S. Okamoto, Y. Izumi, Y. Inoue, and K. Kobayashi, Jpn. J. Appl. Phys., Part 2 38, L1419 (1999).

${ }^{4}$ T. Shosaku, M. Youichi, K. Hiroshi, and S. Hiroshi, J. Electrochem. Soc. 123, 1917 (1976).

${ }^{5}$ C. J. Tong, Z. G. Liu, W. M. Zhao, L. Wang, X. Jiang, Z. Zhang, and S. Xu, Acta Opt. Sin. 19, 402 (1999).

${ }^{6}$ H. S. Bae, T. G. Kim, C. N. Wang, S. Im, J. S. Yun, and J. H. Song, J. Appl. Phys. 91, 4078 (2002).

${ }^{7}$ X. L. Xu, Y. B. Hou, Z. Xu, X. W. Wang, and X. Xu, Jpn. J. Appl. Phys., Part 1 39, 1769 (2000).

${ }^{8}$ T. Matsuzawa, Y. Aoki, N. Takeuchi, and Y. Murayama, J. Electrochem. Soc. 143, 2670 (1996).

${ }^{9}$ C. N. Xu, T. Watanabe, M. Akiyama, and X. G. Zheng, Appl. Phys. Lett. 74, 2414 (1999).

${ }^{10}$ C. N. Xu, X. G. Zheng, M. Akiyama, K. Nonaka, and T. Watanabe, Appl. Phys. Lett. 76, 179 (2000).

${ }^{11}$ H. Matsui, C. N. Xu, T. Watanabe, M. Akiyama, and X. G. Zheng, J. Electrochem. Soc. 147, 4692 (2000).

${ }^{12}$ C. N. Xu, Encyclopedia of Smart Materials; edited by M. Schwarts (Wiley, New York, 2002), pp. 190-201.

${ }^{13}$ Y. Liu and C. N. Xu, J. Phys. Chem. B 107, 3991 (2003).

${ }^{14}$ M. Ohta, M. Maruyama, T. Hayakawa, and T. Nishijo, J. Ceram. Soc. Jpn. 108, 284 (2000).

${ }^{15}$ K. Kato, Y. Ogura, M. Sengiku, K. Shinbo, F. Kaneko, Y. Oda, and K. Yatsui, Jpn. J. Appl. Phys., Part 1 40, 1038 (2001).

${ }^{16}$ K. Kato, I. Tsutai, T. Kamimura, F. Kaneko, K. Shinbo, M. Ohta, and T. Kawakami, J. Lumin. 82, 213 (1999).

${ }^{17}$ Y. S. Park and B. K. Shin, Topics in Physics: Electroluminescence, edited by J. I. Pankove (Springer, New York, 1977), Vol. 17, pp. 133-162; T. Inoguchi and S. Mito, pp. 197-210.

${ }^{18}$ M. Dur, S. M. Goodnick, S. S. Pennathur, J. F. Wager, M. Reigrotzki, and R. Redmer, J. Appl. Phys. 83, 3176 (1998).

${ }^{19}$ C. N. Xu, T. Watanabe, M. Akiyama, and X. G. Zheng, Appl. Phys. Lett. 74, 1236 (1999).

${ }^{20}$ B. P. Chandra, Radiat. Eff. Defects Solids 138, 119 (1996).

${ }^{21}$ C. N. Xu and Y. Liu, US patent, No. 10/025,916 (26 December 2001). 